\title{
CS MULTITRANSITION OBSERVATIONS OF THE CIRCUMNUCLEAR DISK
}

\author{
E. Serabyn \\ California Institute of Technology \\ Downs Laboratory of Physics, 320-47 \\ Pasadena, CA 91125 \\ R. Güsten \\ Max-Planck-Institut für Radioastronomie \\ Auf dem Hügel 69 \\ D-5300 Bonn 1, F.R.G.
}

\author{
N. J. Evans II \\ Astronomy Department \\ University of Texas \\ Austin, TX 78712
}

\begin{abstract}
Three rotational transitions of the CS molecule were observed toward the negative longitude half of the molecular disk which orbits about the galactic center between radii of 2 and $10 \mathrm{pc}$. A comparison of the relative line strengths allows a determination of the radial run of density in the circumnuclear disk. In order to observe all of the transitions with a similar beamsize, the two lower frequency transitions were measured with the IRAM 30-m telescope, and the highest frequency transition with the NRAO 12-m. Table I lists the observing parameters.
\end{abstract}

Figure 1 shows the lineshapes of the $\mathrm{CS} \mathrm{J}=2-1,3-2$, and 5-4 rotational transitions at a position located at the inner edge of the circumnuclear disk. The position is $44^{\prime \prime}$ from the center, along the major axis of the negative longitude, negative velocity $\mathrm{CO}$ emission ridge (Fig. 3). All transitions show the same emission components: negative velocity emission from the circumnuclear disk, and positive velocity emission from the 20 and $50 \mathrm{~km} \mathrm{~s}^{-1}$ clouds which lie along the line-of-sight. The negative velocity emission from the disk has a similar lineshape in all transitions, and, within the noise, line-of-sight absorption is not evident.

The intensities of the three transitions were compared with a "large-velocity-gradient" model for spectral line emission. The predicted line ratios for various gas temperatures and densities are shown in Figure 2.

\section{Table I}

\begin{tabular}{lccccc}
\hline CS line & Freq $(\mathrm{GHz})$ & Telescope & Date & Resolution & Beam \\
\hline $\mathrm{J}=2-1$ & 98 & $30 \mathrm{~m}$ & Aug 86 & $1 \mathrm{MHz}$ & $25^{\prime \prime}$ \\
$\mathrm{J}=3-2$ & 147 & $30 \mathrm{~m}$ & Aug 86 & $1 \mathrm{MHz}$ & $17^{\prime \prime}$ \\
$\mathrm{J}=5-4$ & 245 & $12 \mathrm{~m}$ & Feb 87 & $2 \mathrm{MHz}$ & $25^{\prime \prime}$
\end{tabular}




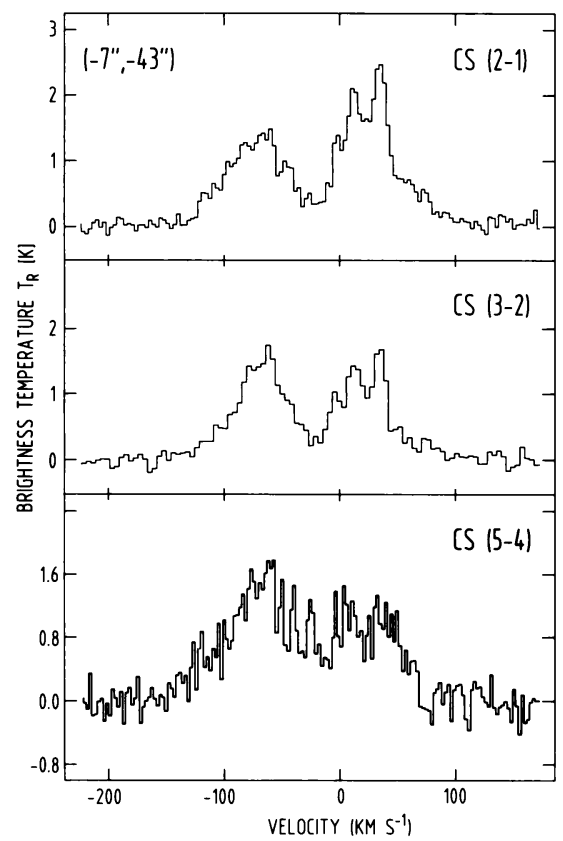

Fig. 1 Spectra of the three CS transitions at $\alpha, \delta$ offsets of $-7^{\prime \prime},-43^{\prime \prime}$ from Sgr A*.

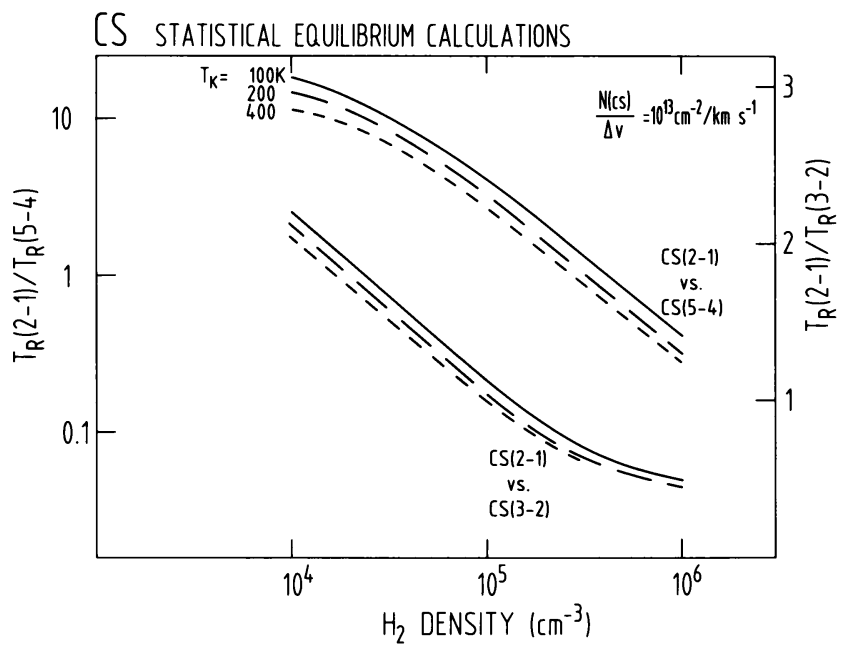

Fig. 2 Predicted CS line ratios vs. density.

Figure 3 shows a composite map of the CO J = 1-0 and HCN J = $1-0$ emission (Serabyn et al. 1986, Güsten $e t$ al. 1987) from the circumnuclear disk. Superposed on this figure are 9 positions at which the CS $\mathrm{J}=3-2$ and $2-1$ transitions were both measured. The densities implied by this pair of lines, assuming $\mathrm{T}=100 \mathrm{~K}$, are given as the upper numbers in the position circles (units are $10^{4} \mathrm{~cm}^{-3}$ ). At 


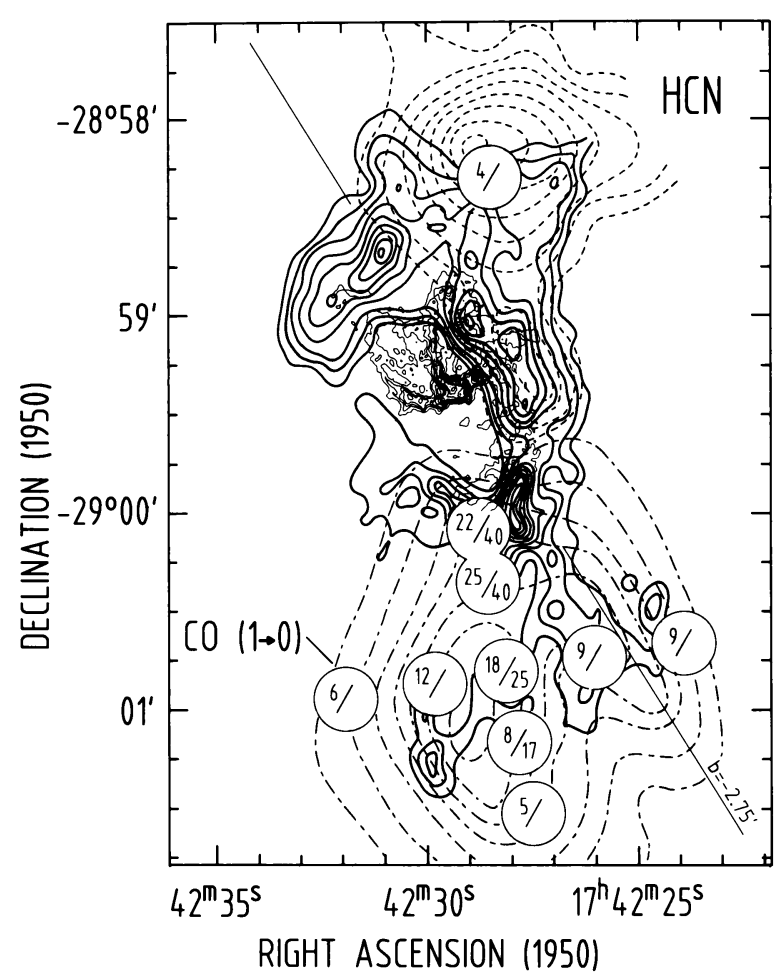

Fig. 3 Composite map of ionized (thin solid contours), $\mathrm{CO} \mathrm{J}=1-0$ (dashed contours), and HCN $J=1-0$ (heavy solid contours) emission from the central few parsecs of our galaxy. The positions at which CS was observed are indicated by the circles. The numbers in the circles give the derived densities $\left(\right.$ in $10^{4} \mathrm{~cm}^{-3}$ ).

four of these positions, the CS 5-4 line was also measured, and the densities implied by the CS $5-4$ to $2-1$ line ratios are shown as the lower numbers in the circles. The observed line ratios indicate that the densities are moderately high everywhere in the disk, about $5 \times 10^{4}-4 \times 10^{5} \mathrm{~cm}^{-3}$. The CS $\mathrm{J}=5-4$ to $\mathrm{J}=2-1$ line ratios give qualitatively similar results to the $\mathrm{J}=3-2$ to $\mathrm{J}=2-1$ line ratios, but the resultant densities are roughly a factor of 2 higher.

The gas density is seen to fall with distance from the center. Given a fixed gas temperature, the densities decrease by a factor of $3-5$ between radii of 2 and $7 \mathrm{pc}$, and by a similar amount along the minor axis cut. A pure temperature falloff with radius can account for only a small fraction of the observed CS $\mathrm{J}=3-2$ to $\mathrm{J}=2-1$ line ratio variations. Harris et al. (1985), and Lugten (1987) find similar excitation variations. Finally, there is no evidence in our data for a very high density phase $\left(n>10^{6} \mathrm{~cm}^{-3}\right)$, required to stabilize clumps against tidal stresses in the central potential.

\section{References}

Güsten, R., Genzel, R., Wright, M. C. H., Jaffe, D. T., and Harris A. I. 1987, Ap. J., 318, 124.

Harris, A. I., Jaffe, D. T., Silber, M., and Genzel, R. 1985, Ap. J., 294, L93.

Lutgen, J. B. 1987, Ph.D. thesis, University of California, Berkeley.

Serabyn, E., Güsten, R., Walmsley, C. M., Wink, J. E., and Zylka, R. 1986, Astr. Ap., 169, 85. 